\title{
Se son rose fioriranno Uno strano caso di nefrolitiasi
}

\author{
Marco Lombardi ${ }^{1}$, Matteo Zoppi ${ }^{2}$, Piero Bruschi ${ }^{3}$, Federico Selvi ${ }^{3}$, Maria Adele Signorini ${ }^{4}$, Roberto Fanelli ${ }^{1}$, \\ Stefano Michelassi ${ }^{5}$ \\ ${ }^{1}$ SOS Emodialisi del Mugello, Ospedale Nuovo del Mugello, Azienda USL Toscana Centro, Firenze \\ ${ }^{2}$ Museo di Storia Naturale dell'Università degli Studi di Firenze, Sezione Mineralogia e Litologia, Firenze \\ ${ }^{3}$ Dipartimento di Scienze delle Produzioni Agroalimentari e dell'Ambiente, Università degli Studi di Firenze, Firenze \\ ${ }^{4}$ Dipartimento di Biologia, Università degli Studi di Firenze, Firenze \\ ${ }^{5}$ SOC Nefrologia e Dialisi, Ospedale S.M. Annunziata, Azienda USL Toscana Centro, Firenze
}

The proof is in the... growing? A peculiar case of kidney stones

A 45-year-old woman affected by MEN-1 syndrome with a history of bilateral nephrolithiasis due to parathyroid adenoma (primary hyperparathyroidism) and celiac disease presented to the nephrologist for recurrent episodes of renal colic and urinary passage of small stones with an unusual morphology despite previous surgical removal of the parathyroid adenoma. A complete diagnostic workup ought to be able to establish the type of stones, but contemporary medicine, with its reliance on protocols, procedures and scientific evidence, may have lost sight of the importance of good communication with the patient.

Key words: Actinidia, Celiac disease, MEN-1, Narrative-based medicine, Nephrolithiasis, Stones

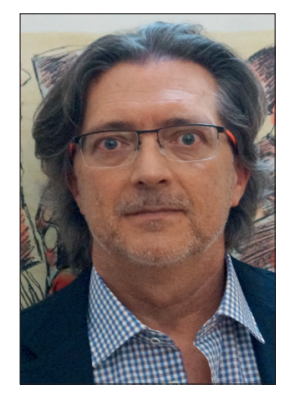

Marco Lombardi

\section{Il caso clinico}

Un anziano medico di Medicina Generale, ormai in pensione, porta all'attenzione del nefrologo la propria figlia di 45 anni (nata nel 1971) perché affetta da nefrolitiasi recidivante.

La paziente ha familiarità per nefrolitiasi calcica, ipertensione arteriosa, osteoporosi, obesità, gotta e malattia cerebrovascolare. All'età di 5 anni era stata affetta da un singolo episodio convulsivo. A 17 anni un traumatismo stradale aveva causato una lesione vertebrale cervicale a livello di C5. A 19 anni le erano stati diagnosticati un gozzo multinodulare pre-tossico e un cistadenoma ovarico destro, quest'ultimo asportato chirurgicamente una

Accepted: June 9, 2017

Published online: September 5, 2017

Indirizzo per la corrispondenza:

Dr. Marco Lombardi

SOS Emodialisi del Mugello

Ospedale del Mugello

Via della Resistenza, 60

50032 Borgo San Lorenzo (FI)

lombardim@tin.it prima volta in quell'anno e nuovamente nove anni più tardi per recidiva. A 21 anni era sopravvenuta la prima colica renale. Le indagini effettuate avevano condotto alla diagnosi di nefrolitiasi radiopaca bilaterale secondaria a iperparatiroidismo primitivo da adenoma paratiroideo inferiore destro. All'età di 30 anni la paziente era stata sottoposta a intervento chirurgico di tiroidectomia totale e paratiroidectomia inferiore destra e da allora era in terapia sostitutiva ormonale tiroidea.

L'intervento sulla paratiroide non aveva interrotto le coliche renali che anzi erano continuate con discreta frequenza, talora tali da richiedere ricovero ma mai procedure urologiche. All'età di 35 anni, in occasione di uno di tali ricoveri, era stata diagnosticata malattia celiaca e la paziente avviata a regime dietetico senza glutine. Rilevate anche osteopenia e carenza di vitamina D. Nei dieci anni successivi erano comparse gotta e ipertensione arteriosa e i vari accertamenti avevano rilevato la presenza di un macroadenoma ipofisario associato a iperprolattinemia, due micronoduli pancreatici con caratteristiche ecografiche compatibili con neoplasie neuroendocrine o intraduttali papillari mucinose (IPMN) e un nodulo (verosimile adenoma) surrenalico destro associato a ipercortisolismo pre-clinico. II corteo tumorale aveva condotto alla diagnosi di adenomatosi endocrina multipla tipo 1 (MEN-1), sindrome ereditaria caratterizzata dalla presenza di tumori delle ghiandole paratiroidi, delle cellule insulari pancreatiche e dell'ipofisi (1). 


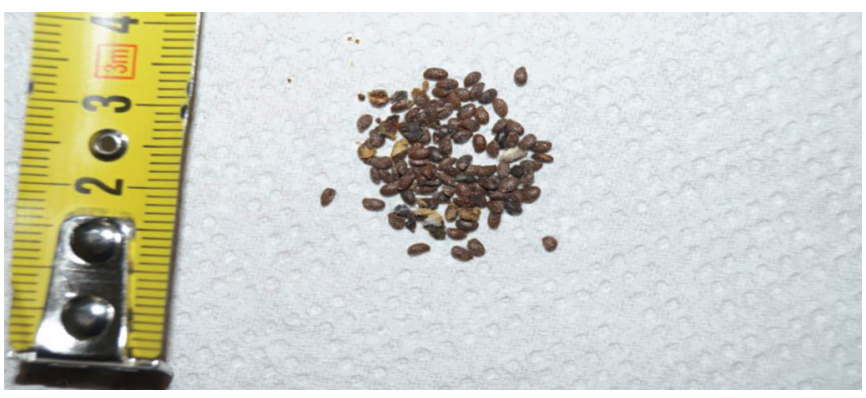

Fig. 1 - Calcoli portati dalla paziente alla prima e unica visita nefrologica.

Alla prima visita nefrologica la paziente appariva in sovrappeso corporeo (BMI 29) e ipertesa (PA 145/95 mm Hg). Riferiva cefalea, diuresi di circa 2 L/die e alvo tendenzialmente diarroico. Aveva una dieta variata, controllata per assenza di glutine, con introito giornaliero di circa 1,5 L di acqua, $100 \mathrm{cc}$ di latte e $150 \mathrm{cc}$ di tè. Assumeva levotiroxina per l'ipotiroidismo (postchirurgico), cabergolina per l'iperprolattinemia, e in modo discontinuo diuretici tiazidici, FANS e vitamina $D$ (calcifediolo). Riferiva persistenza di ricorrenti, modeste, coliche renali e portava una quarantina di calcoli di piccole dimensioni (Fig. 1), eliminati spontaneamente negli ultimi mesi con frequenza quasi settimanale. Una recente analisi chimica di calcoli precedentemente espulsi aveva rilevato la presenza di calcio (+), ossalato $(++)$ e sali ammoniacali $(++)$. L'ecografia addome rilevava microlitiasi caliceale bilaterale e calcificazioni intraparenchimali bilaterali multiple. Gli esami di base mostravano lieve ipercalcemia (calcemia totale $10,7 \mathrm{mg} / \mathrm{dl}, \mathrm{Ca}++1,41$
$\mathrm{mMol} / \mathrm{L})$, ipofosforemia $(2,4 \mathrm{mg} / \mathrm{dl})$ e aumento della fosfatasi alcalina ossea $(30 \mathrm{mcg} / \mathrm{L}) \mathrm{ma}$ non del PTH $(44 \mathrm{pg} / \mathrm{ml})$. Normali sia la funzione renale (creatininemia $0,72 \mathrm{mg} / \mathrm{dl}$ ) che la bicarbonatemia $(28,8 \mathrm{mmol} / \mathrm{L})$. La ricerca di cristalli di cistina all'esame delle urine risultava negativa. Una valutazione metabolica completa per nefrolitiasi sulle urine delle 24 ore non indicava alcuna sovrasaturazione per ossalato di calcio, fosfato di calcio o acido urico (Fig. 2, ultime tre colonne blu a destra). In particolare sulle urine dopo digiuno di 14 ore, calciuria e citraturia risultavano nei limiti quando corretti per l'escrezione di creatinina (rispettivamente 0,1 con v.n. $<0,12$ e 1,38 con v.n. $>0,15$ ) e la concentrazione di acido urico libero nelle urine cadeva in zona di sottosaturazione quando rapportata al $\mathrm{pH}$ urinario. Risultava basso invece l'handling tubulare del fosfato (TmP/GFR 2,1 vs v.n. >2,5). L'attendibilità di tale studio era però inficiata sia dall'assunzione nelle 24 ore precedenti di una quantità di liquidi nettamente superiore rispetto alle normali abitudini (circa 3 vs 1,5 L) sia da una incompleta raccolta delle urine 24 ore (suggerita da una creatininuria di $6,8 \mathrm{mg} / \mathrm{kg} / \mathrm{die}$, nettamente inferiore ai $15-20 \mathrm{mg} / \mathrm{kg} / \mathrm{die}$ attesi per età e sesso) (2). I due "bias" avrebbero potuto giocare entrambi un ruolo nel determinare un risultato falsamente negativo, il primo per eccessiva diluizione urinaria di fattori "litogeni", il secondo per errato calcolo (per difetto) delle quantità degli stessi fattori "litogeni" escreti nelle 24 ore.

All'esame macroscopico tutti i calcoli espulsi dalla paziente avevano caratteristiche omogenee: piccole dimensioni ( $1 \times 2 \mathrm{~mm})$, forma ellissoidale, superficie grossolanamente liscia, color testa di moro, relativamente

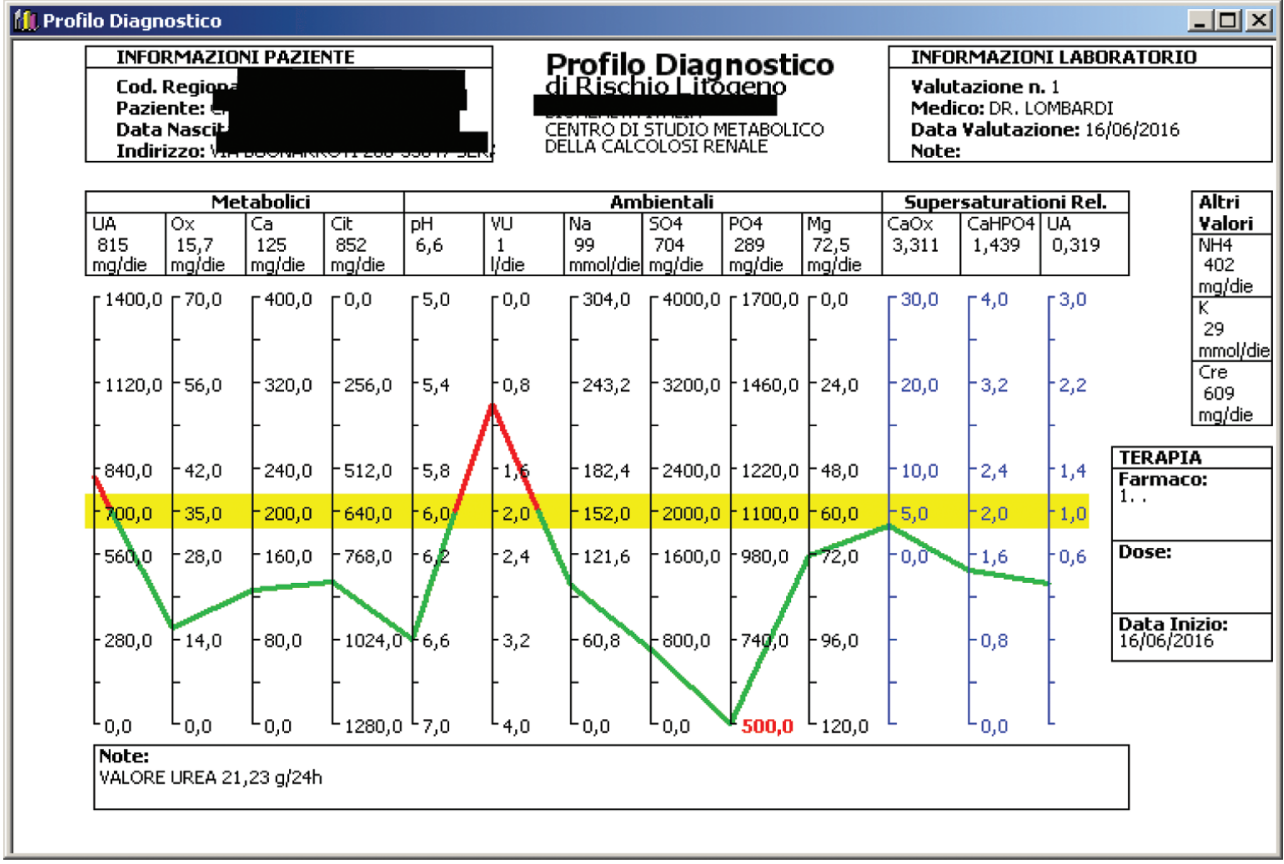

Fig. 2 - Valutazione metabolica urinaria sulle urine delle 24 ore, per la ricerca di sovrasaturazione per i principali componenti dei calcoli renali. 


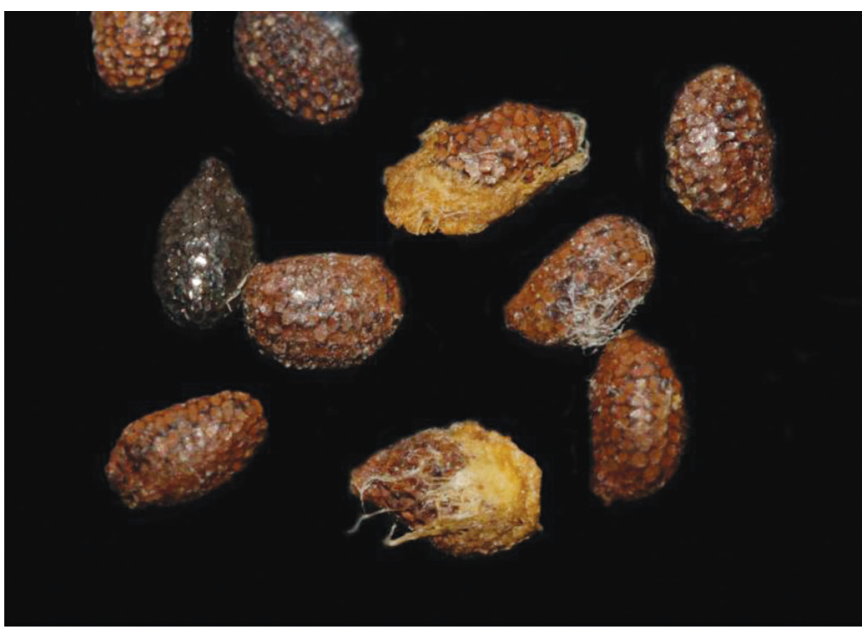

Fig. 3 - Immagine al microscopio ottico, a circa 50 ingrandimenti, dei granuli sottoposti a indagini di spettroscopia micro-Raman.

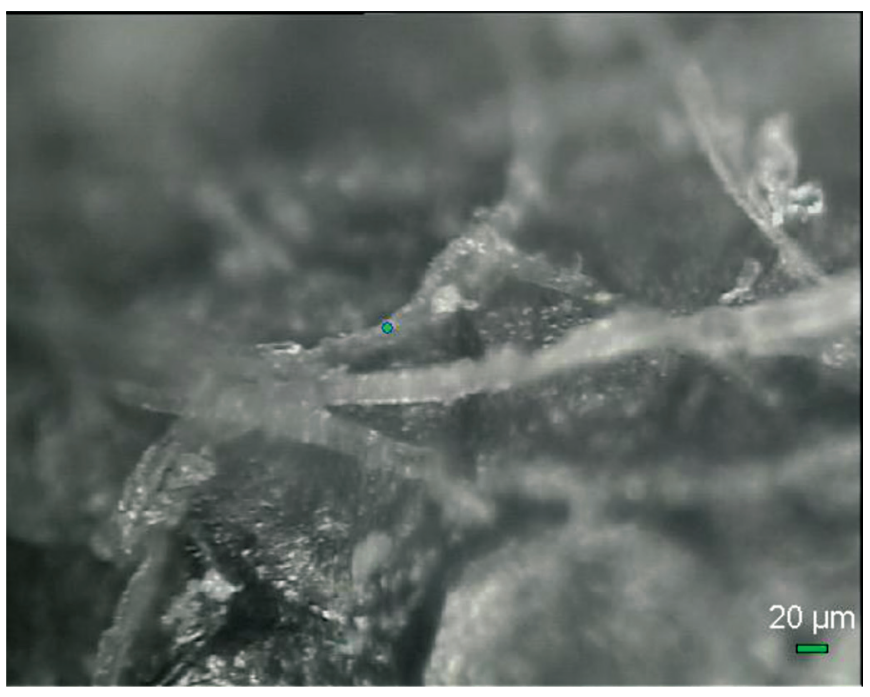

Fig. 4 - Alcuni degli "oggetti" analizzati mostrano la presenza di frammenti e di fibre di colore bianco, semitrasparenti, distribuite non omogeneamente sulla superficie.

friabili (Fig. 2) mentre alla microscopia ottica la loro superficie appariva irregolare e disomogenea (Fig. 3). Infatti l'esame microscopico, effettuato presso il laboratorio di Spettroscopia micro-Raman e micro-FTIR del Museo di Storia Naturale dell'Università degli Studi di Firenze, distingueva una parte esterna di colore marrone-grigiastro, non liscia e omogenea ma costituita da placche pseudoesagonali eventualmente e irregolarmente frammiste a fibre e altri frammenti micrometrici di colore biancastro (Figg. 3, 4), e un nucleo interno, solido ma friabile, di color giallo-ambra, impregnato e circondato da una sostanza viscosa. L'analisi micro-Raman effettuata sia sulla parte esterna che sul nucleo non era in grado di riconoscere sostanze minerali o in generale inorganiche (a eccezione

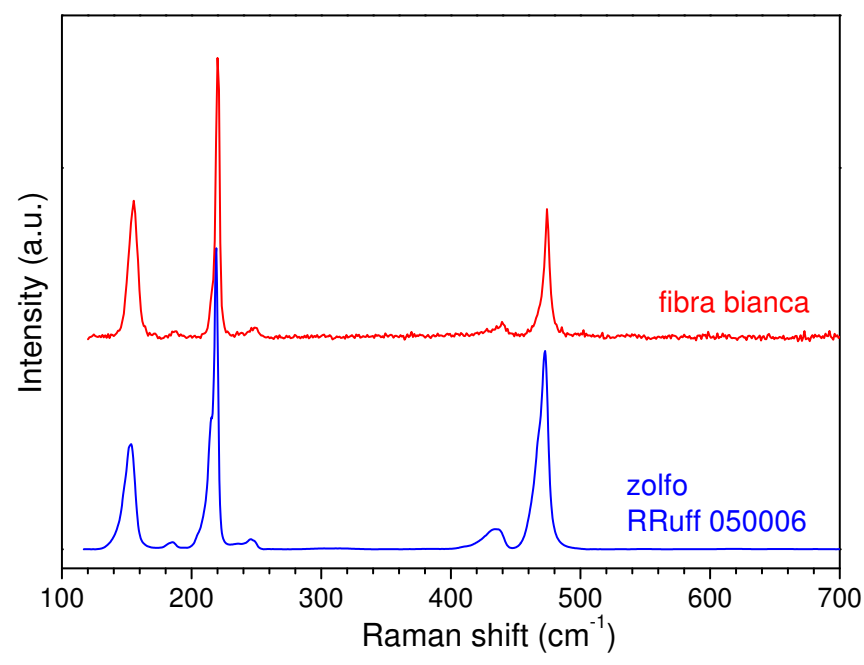

Fig. 5 - Solo in un caso, su un totale di dieci fibre e particelle analizzate, l'analisi micro-Raman ha permesso di rilevare la presenza di zolfo molecolare come unica sostanza inorganica. In rosso lo spettro raccolto, in blu lo spettro di paragone fornito dal database per il riconoscimento delle fasi.

di una singola particella di zolfo molecolare di difficile interpretazione, forse attribuibile a contaminazione, Fig. 5). Diversamente, un gran numero di punti analisi effettuati sulla superficie sia interna che esterna dei calcoli produceva spettri Raman la cui fluorescenza indicava la presenza di sostanze di natura biochimica. Nel reparto di Entomologia della Facoltà di Agraria dello stesso Ateneo veniva escluso potersi trattare di uova o altro materiale proveniente da insetti. Anche l'esame parassitologico e fisico-chimico delle feci della paziente era risultato nella norma. A questo punto, essendo stata esclusa l'appartenenza al regno minerale e animale, non rimaneva che una possibilità per stabilire la natura dei "calcoli" in possesso della paziente. $E$ in effetti, analisi eseguite nei laboratori di Botanica della stessa scuola identificavano con certezza i campioni come semi di piante Angiosperme. Per una definizione specifica del tipo di pianta, impossibile sulla base della sola morfologia dei semi, è stata effettuata una prova di germinazione in ambiente controllato. I semi sono stati sottoposti a un trattamento di chilling $\left(60\right.$ giorni a $3^{\circ} \mathrm{C}$ ) per rimuovere un'eventuale dormienza embrionale e successivamente posti in capsule di germinazione a temperatura ambiente. Dopo 9 giorni alcuni semi hanno germinato e, trasferiti in vaso con substrato standard in serra fredda, hanno prodotto in pochi giorni plantule dicotiledoni con foglioline (Figg. 6 e 7), di forma ovata, con apice acuto e margine dentato. L'analisi combinata della morfologia di semi e plantule ha permesso di stabilire che le piantine appartenevano al genere Actinidia Lindl, verosimilmente alla specie Actinidia chinensis Planch, la pianta che produce il 


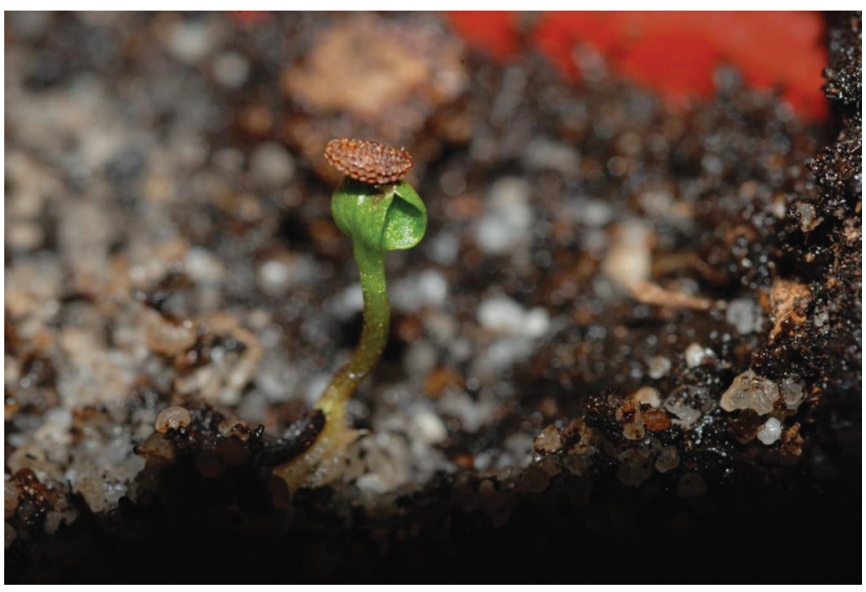

Fig. 6 - Plantule con due piccoli cotiledoni fotosinteticamente funzionali.

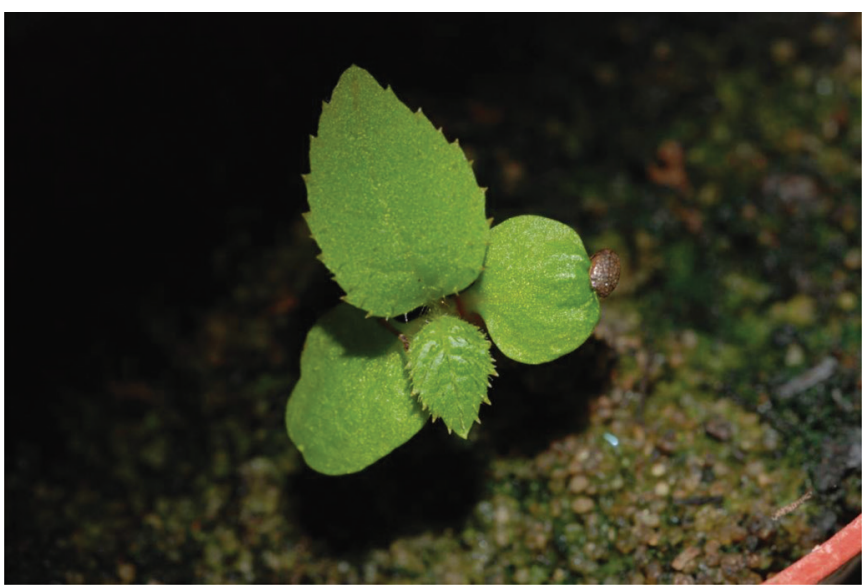

Fig. 7 - Le plantule di Angiosperma dicotiledone hanno tipiche foglioline di forma ovata, con apice acuto e margine dentato.

ben noto frutto del "kiwi". Se son rose, fioriranno. Se son piante di kiwi, ...pure.

Informati dei risultati delle indagini, padre e figlia hanno interrotto gli accertamenti adducendo una serie di problemi personali e familiari di vario genere. A un anno di distanza, contattato telefonicamente, il padre ha riferito la persistente scomparsa sia di coliche che di emissione di "calcoli" di qualunque tipo, già da pochi giorni dopo la prima, e unica, visita nefrologica effettuata dalla paziente.

\section{Discussione}

L'evoluzione finale non deve far deviare dal problema clinico principale. La paziente era realmente affetta da nefrolitiasi bilaterale recidivante a elevata attività metabolica, come confermato dall'esame chimico-fisico di numerosi calcoli emessi anche pochi giorni prima della visita nefrologica (ossalato di calcio), con causa più probabile un iperparatiroidismo primitivo (anche con il reperto ecografico di nefrocalcinosi) nell'ambito di una MEN-1 $(3,4)$. La tendenza all'ipercalcemia negli ultimi esami poteva essere secondaria a una recidiva della patologia paratiroidea, sebbene poco compatibile comunque con i livelli normali di PTH (che avrebbe dovuto essere ricontrollato) e di calciuria (peraltro poco attendibile per l'incompleta raccolta urinaria). Una possibilità alternativa poteva essere una eccessiva assunzione di vitamina $D$, che non è stata inizialmente valutata. Infine, anche la malattia celiaca rientra fra le patologie intestinali in grado di causare calcolosi renale per iper-assorbimento intestinale di ossalato (iperossaluria enterica) (5-8). Purtroppo, il rifiuto della paziente a proseguire gli esami non ha permesso di indagare tutti gli aspetti della sua patologia litiasica.

\section{Conclusioni}

Forse i motivi per cui, nonostante una "vera" calcolosi renale, la paziente abbia finto una condizione patologica ancora peggiore di quella reale, fino a mettere nelle mani del nefrologo semi vegetali come prova di calcoli emessi, nessuno li saprà mai. Ma il caso sembra istruttivo perché pone in rilievo l'importanza per il nefrologo, in casi di nefrolitiasi recidivante, di non fermarsi alla semplice diagnosi di calcolosi (più o meno radiopaca) e indicare mere generiche misure preventive come un maggiore introito idrico e l'assunzione di diuretici tiazidici e/o inibitori della cristallizzazione (citrato di potassio). In questi casi il nefrologo ha il dovere di perseguire sempre le possibili cause della nefrolitiasi del paziente e, quando possibile, indicarne misure preventive specifiche, come ad esempio una adeguata alcalinizzazione delle urine in caso di litiasi urica o l'assunzione di supplementi di calcio e un'acqua a elevato tenore calcico (9) in caso di iperossaluria enterica, ad esempio in corso di celiachia o di altra malattia gastro-intestinale o pancreatica cronica o ancora in casi di dieta ipocalcica da intolleranza al lattosio, per esempio. In quest'ottica i due momenti più significativi del workup diagnostico sono una completa valutazione metabolica (che, come il caso illustra chiaramente, richiede incondizionatamente un'adeguata compliance da parte del paziente) e l'analisi dei calcoli emessi, imprescindibile per decidere il trattamento più adeguato. E proprio la caparbietà del nefrologo nel perseguire fino in fondo la diagnosi è risultata in questo caso il fattore principale che ha condotto a svelare la messinscena della paziente e a suggerirne una condizione psicologica che probabilmente meritava di essere seguita in altro ambiente specialistico.

E però non è tutt'oro ciò che luccica. Se da un lato, sfruttando le proprie competenze cliniche specialistiche, il nefrologo ha ottenuto comunque un successo, arrivando a una, anche sorprendente, conclusione, la perdita del paziente durante il workup diagnostico non può essere av- 
vertita se non come una sconfitta. Avrebbe potuto essere evitata? Forse oggi una Medicina si basa preminentemente su protocolli, procedure ed evidenze scientifiche ha perso di vista il lato comunicativo medico-paziente, tanto caro ai nostri maestri. Forse, impostando il rapporto con paziente e padre in modo diverso, "più olistico", il nefrologo avrebbe potuto prevedere il rischio di perdere la paziente e approcciarsi a lei (e al padre) in modo più "condivisibile". E probabilmente questo diverso comportamento sarebbe risultato vantaggioso per il paziente, se fosse riuscito a evitare il suo rifiuto a proseguire le indagini. In sintesi si è perso oggi di vista quella che più recentemente è stata definita Medicina Narrativa (10), una "metodologia d'intervento clinico-assistenziale basata su una specifica competenza comunicativa" in cui la visita medica prevede uno scambio di narrazioni e una negoziazione di significati che aiuta medico e paziente a sviluppare un'alleanza terapeutica e un percorso di cura che sia da un lato appropriato secondo le indicazioni dell'Evidence Based Medicine ma nello stesso tempo il più possibile personalizzato e quindi più efficace (11).

\section{Disclosures}

Financial support: No financial support was received for this submission. Conflict of interest: The authors have no conflict of interest.

\section{Bibliografia}

1. Lewis CE, Yeh MW. Inherited endocrinopaties: An update. Mol Genet Metab. 2008; 94(3):271-82

2. Gul A, Gilbert SJ, Levey AS. Assessment of renal function. In: Comprehensive Clinical Nephrology, $3^{\text {rd }}$ ed.; Mosby Elsevier 2007; Sect 2; Chapter 3:27-34.

3. Brandi ML, Gagel RF, Angeli A, et al. Guidelines for diagnosis and therapy of MEN type 1 and type 2. J Clin Endocrinol Metab. 2001;86(12):5658-71.

4. Benson L, Ljunghall S, Akerström G, Oberg K. Hyperparathyroidism presenting as the fist lesion in multiple endocrine neoplasia type 1. Am J Med. 1987;82(4):731-7.

5. La Manna A, Apicella A, Cangiano G, Polito C. Urolitiasi del bambino: difficoltà diagnostiche. G Ital Nefrol 2013;30 (2): pii: $\operatorname{gin} / 30.2 .3$.

6. Worcester EM. Stones from bowel disease. Endocrinol Metab Clin North Am. 2002;31:979-99.

7. Robijn S, Hoppe B, Verveat BA, D'Haese PC, Verhulst A. Hyperoxaluria: a gut-kidney axis? Kidney Int. 2011;80; 1146-58.

8. Vitale C. Rodofili A, Bermond F, et al. Diagnostica metabolica della calcolosi renale (Parte II). Giornale di Tecniche $\mathrm{Ne}$ frologiche e Dialitiche. 2017;29(1):24-31.

9. Vitale C. Rodofili A, Bermond F, et al. Diagnostica metabolica della calcolosi renale (Parte I). Giornale di Tecniche Nefrologiche e Dialitiche. 2016;28(4):259-65.

10. Bert G. Medicina Narrativa. Storie e parole nella relazione di cura. Roma: II pensiero Scientifico Editore 2007.

11. Linee di indirizzo per l'utilizzo della Medicina Narrativa in ambito clinico-assistenziale, per le malattie rare e cronico-degenerative, pubblicato nella Collana "I Quaderni di Medicina" de II Sole24Ore Sanità (Allegato al N. 7, 24 feb.-2 mar. 2015). 